\title{
INFLUÊNCIA DA LASERTERAPIA NA DOR E QUALIDADE DE VIDA EM MULHERES COM FIBROMIALGIA
}

\author{
INFLUENCE OF LASER THERAPY ON PAIN AND THE QUALITY OF LIFE IN WOMEN \\ WITH FIBROMYALGIA
}

\author{
Renato Canevari Dutra da Silva ${ }^{1}$ \\ Fabiana Machado Pires ${ }^{2}$ \\ Getúlio Antônio de Freitas Filho ${ }^{3}$ \\ Ana Paula Felix Arantes ${ }^{4}$ \\ Roberto Dias 5 \\ Rejane Maria Cruvinel Cabral ${ }^{6}$
}

Resumo: A fibromialgia é uma síndrome dolorosa, não inflamatória, cujo principal sintoma é a dor crônica musculoesquelética generalizada, causando um impacto negativo na qualidade de vida destes pacientes. $O$ objetivo deste estudo foi investigar a influência da laserterapia na dor e qualidade de vida em mulheres com fibromialgia. Participaram do estudo 9 mulheres com diagnóstico confirmado de fibromialgia, as quais foram submetidas a 10 sessões consecutivas com laser 830 $\mathrm{nm}$ e dosimetria de $5 \mathrm{~J} / \mathrm{cm}^{2}$ aplicado apenas nos tender points dolorosos à palpação digital. Foi realizada a avaliação da intensidade da dor, antes e após a intervenção com laserterapia, utilizando a escala visual analógica (EVA) e, para avaliar qualidade de vida, foram utilizados dois questionários, o Medical Outcomes Study 36-item Short-Form Health Survey (SF-36) e o Fibromyalgia Impact Questionnaire (FIQ), sendo realizados antes e após o tratamento com laserterapia. Na avaliação da intensidade da dor através da EVA, não foi observado diferença significativa após o tratamento $(p=3,03)$. Em relação ao $S F$ 36, houve diferenças estatisticamente significantes do escore geral $(p=0,018)$ e observaram-se diferenças estatisticamente significantes nas variáveis dor $(p=0,032)$ e estado geral de saúde $(p=0,027)$. No FIQ, o escore geral apresentou diferença estatisticamente significante, sendo $(p=0,002)$ e, em relação às variáveis do FIQ, houve diferença estatisticamente significante no bem estar $(p=0,007)$, dificuldade no trabalho $(p=0,048)$, dor $(p=0,000)$, rigidez $(p=0,033)$ e sono $(p=0,021)$. Foi observado que a intervenção fisioterapêutica com laserterapia promove melhora da dor e da qualidade de vida de mulheres com fibromialgia.

Palavras-chave: fibromialgia; dor; qualidade de vida.

Abstract: Fibromyalgia is a painful and non-inflammatory syndrome. The main symptom is widespread and chronic musculoskeletal pain, which is often associated with other physical and emotional components, with negative impact on the quality of life of these patients. Currently, the most accepted hypothesis for the etiology of painful sensation in fibromyalgia is based on changes in the central processing of pain. The aim of this study was to investigate the

\footnotetext{
${ }^{1}$ Mestre em Ciências da Saúde - Universidade de Brasília - UNB, Brasil. Fisioterapeuta do Hospital Municipal de Rio Verde - GO, Brasil e Professor no Instituto de Ensino Superior de Rio Verde - GO - IESRIVER / Faculdade Objetivo, Brasil. E-mail: renatocanevari@yahoo.com.br.

${ }^{2}$ Graduanda em Fisioterapia - IESRIVER. E-mail: fabianampc@hotmail.com.

${ }^{3}$ Mestre em Engenharia Biomédica (Bioengenharia) - Universidade do Vale do Paraíba - Univap, Brasil e Doutorando em Educação Física - Universidade Católica de Brasília - UCB, Brasil. Professor do IESRIVER. E-mail: getulio@faculdadeobjetivo.com.br.

${ }^{4}$ Graduada em Fisioterapia - Universidade Estadual de Goiás - UEG, Brasil e em Biomedicina - Universidade Católica de Goiás - UCG, Brasil. Fisioterapeuta do Hospital Municipal de Rio Verde - GO. E-mail: ana_paula_arantes@hotmail.com. ${ }^{5}$ Mestre em Bioengenharia - Univap. Líder de Fisioterapia da Unimed Rio Verde - GO, Brasil, Professor do IESRIVER, Fisioterapeuta da Clínica de Fisioterapia Andrade e Ltda. e Coordenador de Fisioterapia do Instituto de Previdência e Assistência dos Servidores Municipais de Rio Verde - GO, Brasil - IPARV. E-mail: fisiodias@uol.com.br.

${ }^{6}$ Mestra em Gerontologia - UCB e Doutoranda em Educação Física - UCB. Fisioterapeuta da Associação Beneficente André Luiz - ABAL e Professora do IESRIVER. E-mail: rejanecruvinel@hotmail.com.
} 
influence of laser therapy on pain and the quality of life of women with fibromyalgia. The study included 9 women with a confirmed diagnosis of fibromyalgia, which underwent 10 consecutive sessions with $830 \mathrm{~nm}$ laser and dosimetry of $5 \mathrm{~J} / \mathrm{cm}^{2}$ applied only on painful tender points of digital palpation. We conducted evaluation of pain intensity before and after intervention with laser therapy using a visual analogue scale (VAS). To assess quality of life, two questionnaires were used: the Medical Outcomes Study 36-item Short-Form Health Survey (SF-36) and the Fibromyalgia Impact Questionnaire (FIQ), which were performed before and after the treatment with laser therapy. For the pain intensity evaluated through VAS, no significant difference was observed after the treatment, $(p=3.03)$. Regarding the SF-36, there were statistically significant differences in overall score ( $p=0.018)$, and we observed statistically significant differences in pain variables $(p=0.032)$ and general health status $(p=0.027)$. In $F / Q$, the overall score showed statistically significant difference, as $(p=0.002)$, and in relation to $F I Q$, there was statistically significant difference in well-being $(p=0.007)$, difficulties at work $(p=0.048)$, pain $(p=0.00)$, stiffness $(p=0.033)$, and sleep $(p=0.021)$. We found that physical therapy intervention with laser therapy promotes pain relief and quality of life in women with fibromyalgia.

Keywords: fibromyalgia; pain; quality of life.

\section{INTRODUÇÃO}

A fibromialgia é uma doença reumatológica, que se manifesta no sistema musculoesquelético e é caracterizada por dor crônica e difusa, apresentando sensibilidade principalmente em pontos específicos, conhecidos como Tender Points (SANTOS et al., 2006).

Por ser uma síndrome dolorosa crônica, pode causar um impacto negativo na qualidade de vida desses pacientes, trazendo uma vasta sintomatologia, como dor generalizada, insônia, fadiga, distúrbios de humor, parestesias de extremidades e rigidez matinal (HEYMANN et al., 2010).

A patogênese dessa síndrome ainda não está totalmente esclarecida, porém as maiores evidências apontam para um distúrbio do mecanismo central do controle da dor ou um processamento alterado do sistema nervoso central em resposta a um estímulo nociceptivo (BRAZ et al., 2011).

Os critérios de avaliação, para o diagnóstico da fibromialgia, foram estabelecidos, em 1990, pelo Colégio Americano de Reumatologia (JORGE; TOMIKAWA; JUCÁ, 2007). A estimativa é que $2 \%$ da população mundial seja portadora dessa síndrome, em cuja maior proporção acometida estejam as mulheres (PROVENZA et al., 2004).

Nesse contexto, inúmeros recursos terapêuticos têm sido propostos com o objetivo de melhorar o quadro álgico, eliminar os tender points e melhorar a qualidade de vida desses pacientes.

A laserterapia de baixa intensidade pode trazer benefícios, principalmente no que se refere à diminuição do quadro álgico, resultando em consequente melhora da qualidade de vida desses pacientes (FERREIRA; MARINO; CAVENAGHI, 2011).

Devido à vasta sintomatologia e ao caráter crônico da fibromialgia, é importante encontrar tratamentos efetivos, que minimizem seu impacto no cotidiano desses pacientes. Contudo os benefícios que a laserterapia de baixa intensidade proporciona são evidentes, 
porém os estudos dessa terapia, aplicada à fibromialgia, ainda é restrito, justificando mais estudos visando à sua efetividade e confiabilidade.

O objetivo deste estudo foi verificar a influência do laser de baixa intensidade na dor e qualidade de vida de mulheres com fibromialgia, em que foi verificado alterações na intensidade da dor por meio da Escala Visual Analógica (EVA), e a qualidade de vida por intermédio dos questionários Short Form Health Survey (SF-36) e do Questionário de Impacto da Fibromialgia (FIQ).

A fibromialgia é uma síndrome dolorosa músculo-esquelética crônica, não inflamatória, caracterizada pela presença de dor difusa pelo corpo e sensibilidade exacerbada à palpação de determinados sítios denominados pontos dolorosos, chamados de tender points (SCOTTON et al., 2010).

Em quadros dolorosos de evolução crônica, como a síndrome da fibromialgia, sem patologia estrutural demonstrável, a dor perde a função de alarme e passa a ser o núcleo fundamental do problema determinando um impacto negativo na qualidade de vida desses pacientes (BRAZ et al., 2011).

Segundo Cardoso et al. (2011), a fibromialgia é o segundo distúrbio reumatológico mais comum nos Estados Unidos, afetando, aproximadamente, quatro a seis milhões de americanos.

A prevalência global, nos Estados Unidos, é de 6\% a 15\%, com incidência cinco vezes maior entre as mulheres do que nos homens (JAHAN et al., 2012).

De acordo com Scotton et al. (2010), há predominância do sexo feminino, sendo $80 \%$ a $90 \%$ dos casos, com um pico de incidência entre 30 e 50 anos de idade, podendo manifestar-se em crianças, adolescentes e indivíduos idosos.

A fibromialgia ainda tem sua etiologia desconhecida, embora a fisiopatologia seja baseada em possíveis alterações na concentração de neurotransmissores, no metabolismo muscular, fisiologia do sono, função neuro-hormonal, estado psicológico e fluxo sanguíneo cerebral (SCOTTON et al., 2010).

Braz et al. (2011) dizem que as maiores evidências para a patogênese da fibromialgia apontam para um distúrbio de modulação central da dor ou um processamento alterado do sistema nervoso central em resposta a um estímulo nociceptivo.

Atualmente, estudos sugerem que a fibromialgia tem origem multifatorial, na qual a ansiedade prolongada, estresse emocional, traumas, doenças ocupacionais, interrupção repentina de medicamentos, hipertireoidismo e infecções podem ser fatores de risco para desencadear a doença (FERREIRA; MARINO; CAVENAGHI, 2011).

Segundo Scotton et al. (2010), o sintoma característico da fibromialgia é dor muscular generalizada, frequentemente acompanhada de rigidez, fadiga, anormalidades na qualidade do sono e distúrbios do humor. 
A vasta sintomatologia resulta, frequentemente, na diminuição da funcionalidade, da capacidade de trabalho e na redução da qualidade de vida (RICCI et al., 2010).

Em 1990, foi definido, pelo American College of Rheumatology (ACR), os critérios de classificação da fibromialgia, sendo validados para a população brasileira em 1999 (HAUN et al., 1990).

De acordo com o ACR, o indivíduo deve apresentar dor generalizada crônica e dor em 11 de 18 pontos dolorosos padronizados, os tender points (JORGE; TOMIKAWA; JUCÁ, 2007).

Conforme Miranda e Freixo (2009), a ACR de 1990 estabeleceu que a palpação digital deve ser feita com uma pressão de $4 \mathrm{kgf} / \mathrm{cm}^{2}$.

Provenza et al. (2004) dizem, ainda, que a palpação digital é a técnica mais utilizada, em que a força necessária para tornar pálido o leito ungueal do primeiro dedo é a pressão apropriada nos tender points, para se estabelecer o que constitui um estímulo doloroso.

O tratamento da síndrome de fibromialgia requer uma abordagem multidisciplinar, sendo necessário incluir mudanças no estilo de vida, tratamento não farmacológico e intervenções farmacológicas (BRAZ et al., 2011).

A fisioterapia e reabilitação ou relaxamento podem ser utilizados no tratamento da fibromialgia, dependendo das necessidades de cada paciente (HEYMANN et al., 2010).

Além disso, Ricci (2010) diz que a eletrotermofototerapia pode trazer benefícios aos pacientes fibromiálgicos, sendo utilizadas, como parte do programa global de reabilitação, principalmente em relação ao alívio da dor.

O laser de baixa potência possui efeitos terapêuticos, como a ação anti-inflamatória, a analgesia e a modulação da atividade celular (FERREIRA; MARINO; CAVENAGHI, 2011).

O efeito analgésico do laser é proveniente da inibição da formação do potencial de ação no nervo periférico, afetando a condução do estímulo nervoso, diminuindo ou interrompendo a transmissão dos impulsos evocados dos nociceptores para a medula espinhal (NEIVA et al., 2010).

De acordo com Meireles (2012), a liberação periférica de opióides das células do sistema imunológico, com liberação de $\beta$-endorfina local, é uma das possibilidades para o efeito analgésico do laser de baixa potência.

Um fator importante, para a efetividade do tratamento com o laser, é o número de aplicações, no qual a World Association for Laser Therapy (WALT) recomenda sessões diárias por duas semanas, ou em dias intercalados, por três a quatro semanas (RICCl et al., 2010).

Com isso, a laserterapia de baixa intensidade pode proporcionar a redução do quadro álgico na fibromialgia, podendo desencadear um efeito cascata para a melhora dos demais sintomas (RICCl et al., 2010). 


\section{METODOLOGIA}

Este estudo caracteriza-se como uma pesquisa de campo, na qual foram pesquisadas 20 mulheres com diagnóstico de fibromialgia, segundo os critérios do ACR, provenientes da cidade de Rio Verde, Goiás.

Foi avaliada a influência da laserterapia de baixa intensidade na dor em mulheres com fibromialgia por meio da Escala Visual Analógica (EVA) e a qualidade de vida foi avaliada por intermédio de dois questionários, o Medical Outcomes Study 36-item Short-Form Health Survey (SF-36) e o Fibromyalgia Impact Questionnaire (FIQ).

O tratamento com laserterapia de baixa intensidade teve duração de 10 sessões consecutivas, ou seja, foi aplicado diariamente.

A seleção foi realizada segundo os seguintes critérios inclusão: 1) Mulheres; 2) Idade entre 25 a 60 anos; 3) Residentes na cidade de Rio Verde; 4) Diagnóstico confirmado de fibromialgia segundo o ACR de1990; 5) Apresentar nível cognitivo suficiente para entender os procedimentos e acompanhar as orientações dadas; 6) Consentir em participar do estudo de forma voluntária e assinar o Termo de Consentimento Livre e Esclarecido (TCLE).

Os critérios de exclusão foram: 1) Menores de 25 anos e maiores de 60 anos; 2) Não estar de acordo com os critérios de diagnóstico do ACR; 3) Não consentir com a pesquisa; 4) Doenças neurológicas; 5) Predisposição ao câncer; 6) Possuir mais de uma falta no programa; 7) Estar realizando tratamentos farmacológicos específico para fibromialgia.

A amostra inicial foi estabelecida com número de 20 mulheres, devido à disponibilidade dos pesquisadores e, acima de tudo, a dificuldade de encontrar mulheres com diagnóstico de fibromialgia confirmado com laudo médico.

A partir do total de 20 mulheres incluídas neste estudo, houve perda na amostra de cinco (5) mulheres por apresentarem uma ou mais condições observadas no critério de exclusão e seis (6) mulheres por abandonarem o tratamento. A análise estatística foi baseada nos 9 sujeitos que concluíram o tratamento com laserterapia.

Este trabalho foi aprovado pelo Comitê de Ética em Pesquisa da FESURVUniversidade de Rio Verde, Goiás, com protocolo de aprovação 024/2013.

Esta pesquisa foi desenvolvida no laboratório de cinesioterapia e eletrotermofototerapia do curso de Fisioterapia, no Instituto de Ensino Superior de Rio Verde - IESRIVER no estado de Goiás.

Os procedimentos seguiram os seguintes passos: foi realizada uma reunião com todas as mulheres, na qual foram feitos esclarecimentos sobre a realização da pesquisa e, em seguida, orientações sobre a síndrome de fibromialgia.

Posteriormente, foi entregue às mulheres o Termo de Consentimento Livre Esclarecido, para que pudessem ler e assinar, em duas vias, assim autorizando sua participação na pesquisa. 
As mulheres foram submetidas ao protocolo de avaliação da fibromialgia, constando os dados pessoais, idade, peso, profissão, estado civil, a história da dor, tempo do diagnóstico da fibromialgia, confirmado por um médico reumatologista, queixa principal, localização da dor, número de tender points dolorosos à palpação, intensidade da dor, sintomas relacionados, uso de medicamentos e o tempo de uso dos medicamentos.

A escala visual analógica de dor e os questionários de qualidade de vida foram aplicados antes da intervenção com laser e ao término do programa de tratamento, ou seja, após a décima sessão.

O equipamento de laser, utilizado na pesquisa, foi da marca IBRAMED, modelo LASERPULSE, de Arsenieto de Gálio e Alumínio GaAIAs, com o comprimento de onda em $830 \mathrm{~nm}$, enquadrando no espectro eletromagnético de luz não visível ou infravermelho próximo. Cabe ressaltar que o aparelho foi calibrado antes do início do estudo, uma vez que trata-se de um equipamento que foi adquirido, exclusivamente, para a realização deste trabalho, sendo utilizado com a calibração de fábrica.

A potência de saída do laser foi de $30 \mathrm{~mW}$, a área do feixe correspondeu a $0,11600 \mathrm{~cm}^{2}$ e a densidade de energia utilizada foi $5 \mathrm{~J} / \mathrm{cm}^{2}$.

O tempo gasto por ponto foi de 20 segundos, sendo irradiado, pontualmente, nos tender points dolorosos à palpação digital, com duração de 10 dias consecutivos.

As pacientes foram submetidas à higienização prévia com aplicação de algodão embebido com álcool concentrado $70 \%$ nos tender points e o laser foi aplicado de forma pontual, com a caneta em 90 graus, em todos os tender points dolorosos.

A caneta do laser foi envolvida com plástico de PCV, para aplicação, o qual foi substituído a cada paciente.

A análise estatística foi realizada pelo software BioEstat 4.0. Os dados coletados foram tabulados no programa Excel, no qual foi realizada a análise descritiva. Para a comparação das variáveis, foi utilizado o teste t de Student. Foi aceito como, estatisticamente, significante resultados com $p \leq 0,05$.

\section{RESULTADOS}

Concluíram o estudo nove mulheres com síndrome da fibromialgia que foram submetidas ao tratamento com laserterapia de baixa intensidade. O período da coleta dos dados iniciou-se em 08 de setembro de 2012 e terminou em 20 de setembro de 2012.

A Figura 1 demonstra quanto ao uso de medicamentos das mulheres fibromiálgicas, sendo o analgésico o mais prevalente com $88,88 \%$, o antidepressivo com $66,66 \%$ e o relaxante muscular com $44,44 \%$. Foi observado que, além do uso de medicamentos para o tratamento específico da fibromialgia, $88,88 \%$ dessas também faziam uso de outros tipos de medicamentos para outras comorbidades associadas à fibromialgia. 


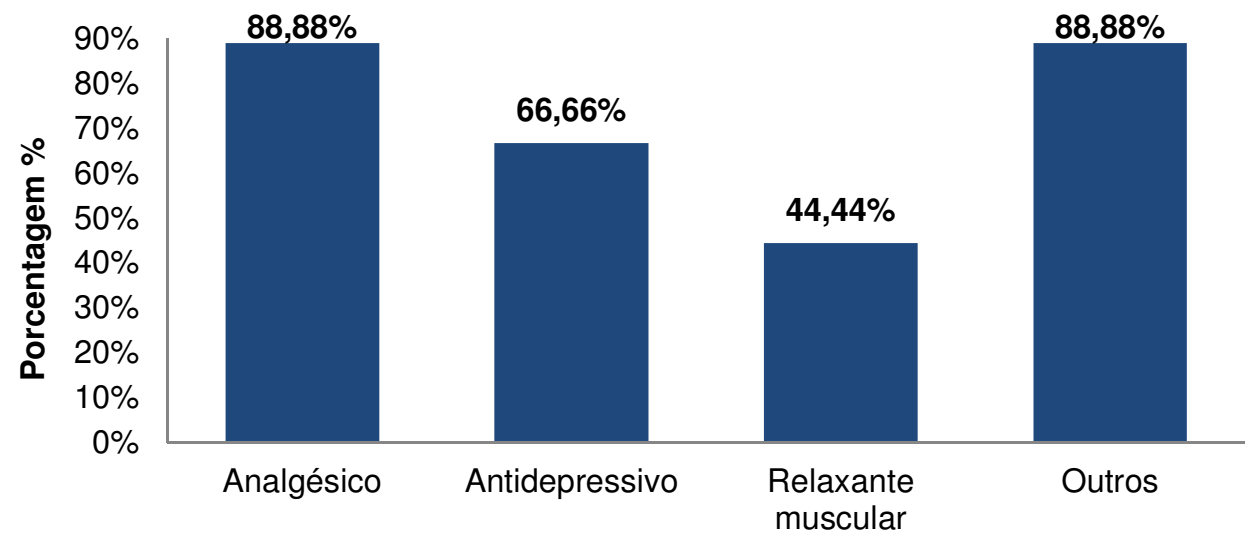

Figura 1 - Uso de medicamentos para o tratamento da fibromialgia.

Ao analisar o número de tender points dolorosos à palpação pré e pós o tratamento com laserterapia de baixa intensidade, observa-se, na Tabela 1, a média e o desvio padrão das pacientes, em que podemos, também, observar que houve diferença estatisticamente significativa dos tender points $(p=0,002)$.

Tabela 1 - Tender points dolorosos pré e pós-tratamento com laserterapia

\begin{tabular}{llll}
\hline Tender Point & Média & DP & P \\
\hline Pré & 17,77 & $\pm 0,66$ & $0,002^{*}$ \\
Pós & 15,44 & $\pm 1,81$ & \\
\hline
\end{tabular}

*diferença estatisticamente significativa $(p \leq 0,05)$.

A Figura 2 mostra os sintomas mais comuns associados à fibromialgia, os resultados apresentados mostram que a prevalência de dor e fadiga se destaca, estando presentes em $99,99 \%$ das mulheres com fibromialgia e rigidez, com $88,88 \%$.

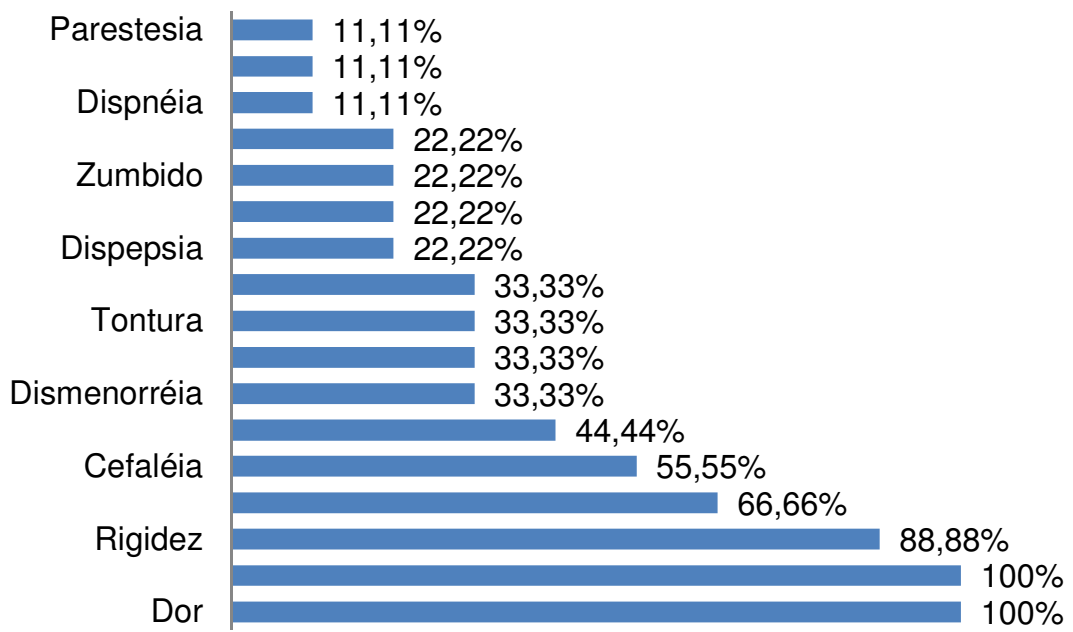

Figura 2 - Sintomas relacionados à fibromialgia da amostra. 


\subsection{Escala Visual Analógica (EVA)}

A Figura 3 apresenta os resultados da EVA, escala visual analógica pré e pós-tratamento com laserterapia nas mulheres fibromiálgicas do presente estudo e percebe-se que houve redução no valor bruto obtido pela EVA, antes e após a intervenção, em todas as participantes do estudo, fato que permite inferir melhora do quadro álgico.

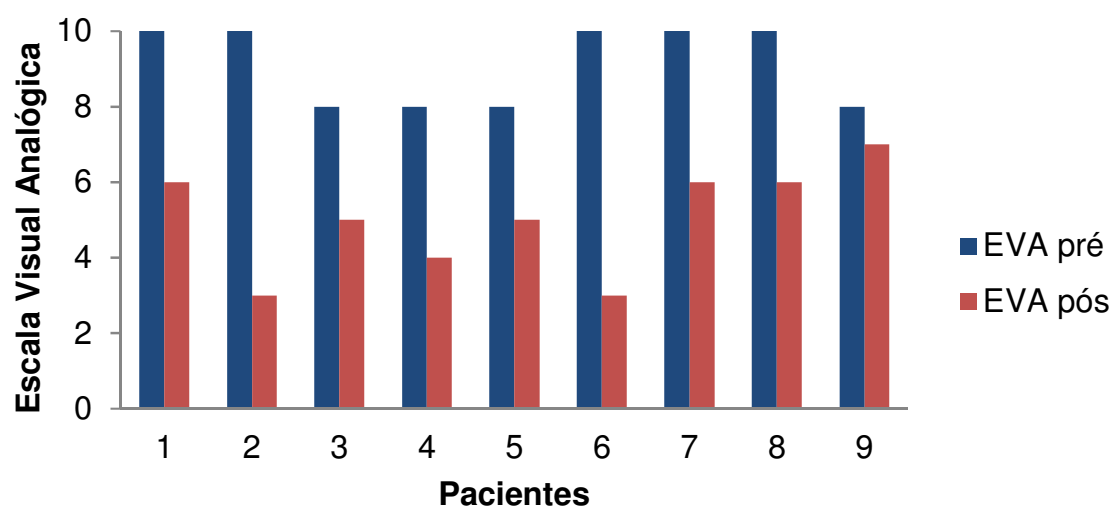

Figura 3 - Resultado da escala visual analógica de cada paciente.

Na Tabela 2, é apresentado o resultado da escala visual analógica, demonstrando a média e o desvio padrão pré e pós-tratamento, em que podemos observar que não houve diferença, estatisticamente, significativa, sendo o $p=3,03$.

Tabela 2 - Escala visual analógica pré e pós-tratamento

\begin{tabular}{llll}
\hline EVA & Média & Desvio Padrão & p \\
\hline Pré & 9,11 & $\pm 1,05$ & \\
Pós & 5,00 & $\pm 1,41$ & 3,03 \\
\hline
\end{tabular}

\subsection{Medical Outcomes Study 36-item Short-Form Health Survey (SF-36)}

Em relação à qualidade de vida, a Tabela 3 mostra os dados avaliados pelo questionário Medical Outcomes Study 36-item Short-Form Health Survey (SF-36). É importante lembrar que o questionário SF-36 varia de 0 a 100, e quanto maior o escore melhor é a qualidade de vida. Os dados estão detalhados por domínio pré e pós-tratamento e são apresentados na tabela a seguir, apresentando as médias, desvio padrão e $p$ de significância. Observa-se que houve melhora significante nas categorias: dor $(p=0,032)$ e estado geral de saúde $(p=0,027)$. Em geral, houve melhora da qualidade de vida, uma vez que a média do escore geral do SF36 , antes e após, apresentou diferença estatisticamente significativa com $p=0,018$. 
Tabela 3 - Resultados do SF-36 antes e após intervenção

\begin{tabular}{lccccc}
\hline \multicolumn{1}{c}{ Domínio } & Média pré & DP & Média pós & DP & p \\
\hline Capacidade funcional & 43,33 & $\pm 22,77$ & 51,11 & $\pm 20,73$ & 0,459 \\
Aspectos físicos & 19,44 & $\pm 32,54$ & 38,88 & $\pm 46,95$ & 0,322 \\
Dor & 24,44 & $\pm 11,51$ & 41,33 & $\pm 18,38$ & $0,032^{*}$ \\
Estado geral de saúde & 37,55 & $\pm 24,55$ & 51,22 & $\pm 26,25$ & $0,027^{*}$ \\
Vitalidade & 35,00 & $\pm 21,50$ & 45,00 & $\pm 26,80$ & 0,395 \\
Aspectos sociais & 48,61 & $\pm 22,04$ & 55,55 & $\pm 24,29$ & 0,534 \\
Aspecto emocional & 29,44 & $\pm 38,72$ & 40,66 & $\pm 49,33$ & 0,598 \\
Saúde mental & 47,11 & $\pm 21,70$ & 56,00 & $\pm 22,44$ & 0,405 \\
Escore geral & 35,61 & $\pm 9,93$ & 47,46 & $\pm 6,85$ & $0,018^{*}$ \\
\hline
\end{tabular}

${ }^{*}$ diferença estatisticamente significativa $(p \leq 0,05)$.

\subsection{Fibromyalgia Impact Questionnaire (FIQ)}

Os resultados apresentados, a seguir, na Tabela 4, são do questionário de impacto da fibromialgia FIQ. Os resultados são apresentados comparando-se a evolução pré e póstratamento com laserterapia. Observa-se que, após o tratamento as mulheres obtiveram melhora, estatisticamente, significante, nos domínios: bem estar ( $p=0,007)$, dificuldade no trabalho $(p=0,048)$, dor $(p=0,000)$, rigidez $(p=0,033)$ e sono $(p=0,021)$. Em geral, houve melhora da qualidade de vida, uma vez que a média do escore geral do FIQ, antes e após, apresentou diferença, estatisticamente, significativa com $p=0,002$.

Tabela 4 - Resultados do FIQ pré e pós-tratamento

\begin{tabular}{lrrrrc}
\hline Domínio & Média pré & DP & Média pós & DP & Valor de $\mathrm{p}$ \\
\hline Capacidade funcional & 3,76 & $\pm 2,69$ & 2,91 & $\pm 2,25$ & 0,494 \\
Bem estar & 7,78 & $\pm 1,90$ & 4,60 & $\pm 2,45$ & $0,007^{*}$ \\
Faltas no trabalho & 3,49 & $\pm 3,04$ & 2,38 & $\pm 3,35$ & 0,471 \\
Dificuldade no trabalho & 6,77 & $\pm 2,68$ & 4,22 & $\pm 2,38$ & $0,048^{*}$ \\
Dor & 8,77 & \pm 1.30 & 5,44 & $\pm 2,06$ & $0,000^{*}$ \\
Fadiga & 7,33 & $\pm 2,39$ & 5,33 & $\pm 2,50$ & 0,102 \\
Rigidez & 7,22 & $\pm 2,48$ & 4,77 & $\pm 1,92$ & $0,033^{*}$ \\
Sono & 7,88 & $\pm 2,31$ & 5,33 & $\pm 1,93$ & $0,021^{*}$ \\
Ansiedade & 6,33 & $\pm 3,35$ & 3,77 & $\pm 2,53$ & 0,087 \\
Depressão & 6,00 & $\pm 3,27$ & 3,66 & $\pm 2,54$ & 0,111 \\
Escore geral & 6,53 & $\pm 1,72$ & 4,24 & $\pm 1,05$ & $0,002^{*}$ \\
\hline
\end{tabular}

${ }^{*}$ diferença estatisticamente significativa $(p \leq 0,05)$.

\section{DISCUSSÃO}

Os dados de literatura mostram que o indivíduo portador de fibromialgia apresenta dor difusa pelo corpo e sensibilidade exacerbada à palpação de determinados pontos chamados 
tender points (SCOTTON et al., 2010). Estudos demonstram que a laserterapia de baixa intensidade pode beneficiar esses pacientes, devido seu efeito terapêutico, como ação antiiflamatória, analgésica e modulação da atividade celular, sendo recomendada, principalmente, para o alívio da dor (FERREIRA; MARINO; CAVENAGHI, 2011). Portanto, a utilização de laserterapia pode trazer benefício adicional para a diminuição da intensidade da dor e, consequentemente, trazer melhora da qualidade de vida dessas pacientes.

Este estudo verificou a eficácia do tratamento com laserterapia de baixa intensidade, avaliando a dor e qualidade de vida pré e pós-tratamento. Os benefícios encontrados foram diminuição da intensidade da dor, representada na Figura 3, e melhora da qualidade de vida, apresentada na Tabela 3 e Tabela 4.

No tratamento farmacológico da fibromialgia, são utilizados os antidepressivos, os moduladores dos canais de cálcio, relaxantes musculares e analgésicos, entre outros fármacos (BRAZ et al., 2011). Quanto ao uso de medicamentos prevalente entre as mulheres fibromiálgicas neste estudo, o analgésico se destaca com $88,88 \%$, o antidepressivo com $66,66 \%$ e o relaxante muscular com $44,44 \%$. Foi observado que além do uso de medicamentos para o tratamento específico da fibromialgia, $88,88 \%$ das mulheres também fazem uso de outros tipos de medicamentos para outras comorbidades associadas à fibromialgia.

As participantes da pesquisa apresentaram vários sintomas associados à fibromialgia, sendo que os mais prevalentes foram à dor e fadiga, destacando-se em 100\% das mulheres com fibromialgia, e rigidez com 88,88\%. Segundo Ricci et al. (2010), a fibromialgia possui uma vasta sintomatologia que resulta, frequentemente, na diminuição da funcionalidade, da capacidade de trabalho e na redução da qualidade de vida.

O tempo de tratamento proposto, neste estudo, foi de 10 dias consecutivos, que foram aplicados diariamente, pois, segundo Ricci et al. (2010), um fator importante para a efetividade do tratamento com o laser é o número de aplicações, sendo que a World Association for Laser Therapy (WALT) recomenda sessões diárias por duas semanas ou em dias intercalados por três a quatro semanas.

Foi utilizado a densidade de energia de $5 \mathrm{~J} / \mathrm{cm}^{2}$, em todos os tender points dolorosos, com duração de 20 segundos, em cada tender point. De acordo com Ricci et al. (2010), vale ressaltar que os efeitos dessa terapia são doses dependentes, e elas variam, amplamente, de 1 a $23 \mathrm{~J} / \mathrm{cm}^{2}$. Nesta pesquisa, foi utilizada a mesma dose do laser em todos os tender points e para todos os sujeitos, mesmo reconhecendo que existem variações das camadas teciduais entre os indivíduos. Contudo houve melhora significativa na diminuição do número de tender points dolorosos à palpação digital, sendo, estatisticamente, significativo $(p=0,002)$. Em uma pesquisa, observaram que pacientes fibromiálgicos possuem pequena quantidade de colágeno intramuscular, com isso, podendo deixar o músculo mais propenso à microlesões, resultando em sinais não específicos de patologia (GRONEMANN et al., 2004).

Segundo Ruaro (2006), na laserterapia, as densidades de energia recomendada para 
promover incremento vascular aumentam de fibroblastos e, consequentemente, de fibras de colágena deve se situar entre 1 a $5 \mathrm{~J} / \mathrm{cm}^{2}$. A dose utilizada, nesta pesquisa, foi de $5 \mathrm{~J} / \mathrm{cm}^{2}$, estando no parâmetro recomendado por esse autor, obtendo melhora clínica significante.

Com isso a aplicação do laser foi realizada em todos os tender points dolorosos à palpação, sendo que a localização destes foi por meio da palpação digital, que é a técnica mais utilizada, em que a força necessária, para tornar pálido o leito ungueal do primeiro dedo, é a pressão apropriada para se estabelecer o que constitui um estímulo doloroso (PROVENZA et al., 2004).

A escala visual analógica foi um instrumento eficaz e confiável para avaliar a intensidade da dor, em que, por meio da comparação, pré e pós-tratamento, percebe-se que houve melhora da intensidade da dor, a qual ocorreu gradualmente. Segundo Meireles et al. (2012), a redução da dor causada pelo laser pode vir de efeitos causados pela liberação periférica de opióides das células do sistema imunológico, com liberação de $\beta$-endorfina local.

$\mathrm{Na}$ realização deste estudo, constatou-se que as publicações sobre o tratamento com laser trazem resultados conflitantes. Não há uma metodologia similar sobre os parâmetros e muitos trabalhos não informam todos os parâmetros usados, proporcionando grande dificuldade de comparação dos resultados. A dosimetria usada no tratamento da síndrome da fibromialgia ainda é restrita, mas, mesmo assim, na prática clínica o laser é uma modalidade muito utilizada.

Diante desse fato, sugere-se a realização de novas pesquisas com mais rigor metodológico proposto para a fibromialgia, para obter uma prática baseada em evidências de forma efetiva, segura e adequada. Outro aspecto importante é a realização de novos estudos, com maior número de sujeitos e maior número de sessões, variação de todos os parâmetros.

\section{CONCLUSÃO}

As mulheres portadoras de fibromialgia submetidas ao tratamento com laserterapia de baixa potência, com dose de $5 \mathrm{~J} / \mathrm{cm}^{2}$, e comprimento de onda $830 \mathrm{~nm}$, apresentaram melhora significativa da intensidade da dor e da qualidade de vida, entretanto, qualquer conclusão seria precipitada quanto à dose ideal, efeitos adversos, e população alvo. Isso devido à pequena quantidade de sujeitos expostos à terapia $(n=9)$ e, também, não houve seguimento deste estudo, ou seja, em longo prazo.

\section{REFERÊNCIAS}

BRAZ, A. S. et al. Uso da terapia não farmacológica, medicina alternativa e complementar na fibromialgia. Revista Brasileira de Reumatologia, Paraíba, v. 51, n. 3, pp. 269-82, mar. 2011.

CARDOSO, F. S. et al. Avaliação da qualidade de vida, força muscular e capacidade funcional em mulheres com fibromialgia. Revista Brasileira de Reumatologia, São Paulo, v. 51, n. 4, 
pp. 338-50, fev. 2011.

FERREIRA, L. L.; MARINO, L. H. C.; CAVENAGHI, S. Recursos eletrotermofototerapêuticos no tratamento da fibromialgia. Revista Dor, São Paulo, v. 12, n. 3, pp. 256-60, 2011.

GRONEMANN, S. T. et al. Collagen and muscle pathology in fibromyalgia patients. Rheumatology, Oxford, v. 43, pp. 27-31, 2004.

HAUN, M. V. A. et al. Validação dos critérios do Colégio Americano de Reumatologia (1990) para classificação da fibromialgia, em uma população brasileira. Revista Brasileira de Reumatologia, v. 39, n. 4, pp. 221-30, jul.-ago.1990.

HEYMANN, R. et al. Consenso brasileiro do tratamento da fibromialgia. Revista Brasileira de Reumatologia, São Paulo, v. 50, n. 1, pp. 56-60, 2010.

JAHAN, F. et al. A syndrome da fibromialgia: uma visão geral de diagnóstico, fisiopatologia e gestão. Med. J., Oman, v. 27, n. 3, pp. 192-195, may. 2012.

JORGE, L. L.; TOMIKAWA, L. C. O.; JUCÁ, S. S. H. Efeito de um programa de reabilitação multidisciplinar para homens portadores de fibromialgia: estudo aleatorizado controlado. ACTA FISIATR, v. 14, n. 4, pp. 196-203, jul. 2007.

MEIRELES, A. et al. Avaliação do papel de opióides endógenos na analgesia do laser de baixa potência, 820 nm, em joelho de ratos Wistar*. Revista Dor, São Paulo, v. 13, n. 2, pp. 152-5, abr.-jun. 2012.

MIRANDA, E. F.; FREIXO, M. R. Aplicação dos critérios de classificação para pacientes com hipótese de fibromialgia. Consciência e Saúde, São Paulo, v. 8, n. 3, pp. 467-475, ago. 2009.

NEIVA. F. C. et al. Analgesia com laser terapêutico após tonsilectomia. Revista Paulista de Pediatria, São Paulo, v. 28, n. 3, p. 322-8, jan. 2010.

PROVENZA, J. R. et al. Fibromialgia. Revista Brasileira de Reumatologia, São Paulo, v. 44, n. 6; pp. 443-449, dez. 2004.

$\mathrm{RICCl}, \mathrm{N}$. et al. A utilização dos recursos eletrotermofototerapêuticos no tratamento da síndrome da fibromialgia: uma revisão sistemática. Revista Brasileira de Fisioterapia, São Carlos, v. 14, n. 1, pp. 1-9, jan.-fev. 2010.

RUARO, J. A. Análise da laserterapia de baixa potência no tratamento de pacientes portadores de fibromialgia. 2006. 107f. Dissertação (Mestre em Engenharia Biomédica) Instituto de Pesquisa e Desenvolvimento, Universidade do Vale do Paraíba, São José dos Campos, SP, 2006.

SANTOS, A. M. B. et al. Depressão e qualidade de vida em pacientes com fibromialgia. Revista Brasileira de Fisioterapia, São Carlos, v. 10, n. 3, pp. 317-324, jul.-set. 2006.

SCOTTON, S. S. et al. Como diagnosticar e tratar fibromialgia. Revista Brasileira de

Medicina, $\quad$ v. 67, $\quad$ n. 7 , jul. 2010. Disponível em: $<\mathrm{http}$ ://www.moreirajr.com.br/revistas.asp?fase=r003\&id_materia=4324>. Acesso em: 8 nov. 2012. 